\title{
The Influence of Chinese College Students' Physical Exercise on Life Satisfaction: The Chain Mediation Effect of Core Self-evaluation and Positive Emotion
}

OPEN ACCESS

Edited by:

Petar M. Mitic,

University of Nis, Serbia

Reviewed by: Antonio Hernández-Mendo, University of Malaga, Spain Maja Batez,

University of Novi Sad, Serbia

${ }^{*}$ Correspondence:

Bo Jiang

jbo@swu.edu.cn

Specialty section:

This article was submitted to

Health Psychology,

a section of the journal

Frontiers in Psychology

Received: 23 August 2021 Accepted: 02 November 2021 Published: 23 November 2021

Citation:

Liu F, Zhu Z and Jiang B (2021) The

Influence of Chinese College Students' Physical Exercise on Life Satisfaction: The Chain Mediation

Effect of Core Self-evaluation and

Positive Emotion.

Front. Psychol. 12:763046.

doi: 10.3389/fpsyg.2021.763046

\author{
Feiyang Liu ${ }^{1}$, Zhengguang Zhu $^{2}$ and Bo Jiang ${ }^{3 *}$ \\ ${ }^{1}$ Physical Education College, Southwest University, Chongqing, China, ${ }^{2}$ Faculty of Psychology, Southwest University, \\ Chongqing, China, ${ }^{3}$ School Physical Education Development Institute, Southwest University, Chongqing, China
}

Physical exercise is an important way for college students to maintain their physical health, and life satisfaction is one of the important indicators of college students' mental health. Therefore, this study aims to explore the relationship between physical exercise and life satisfaction of college students. Additionally, we also seek to demonstrate the chain mediating effects of core self-evaluation and positive emotion on this relationship. A total of 794 Chinese college students, 324 men and 470 women, participated in the study. The participants were $17-25$ years old $(M=19.96 \pm 1.54)$. They completed the Exercise Adherence Questionnaire, Core Self-evaluation Scale, Positive Affect and Negative Affect Scale, and Satisfaction with Life Scale. Results showed a strong positive relationship between physical exercise and life satisfaction and verified the mediating effect of core self-evaluation and positive emotion on this relationship. The results also confirmed the chain mediating model between physical exercise, core self-evaluation, positive emotion, and life satisfaction. It enlightens us that we should pay more attention to the organic combination of students' physical activities and mental health education.

Keywords: physical exercise, core self-evaluation, positive emotion, life satisfaction, college students

\section{INTRODUCTION}

As the backbone of social development, college students' physical and mental health is very important. In health studies, life satisfaction is an individual's perception and evaluation of his or her overall life quality (Dew and Huebner, 1994), which is an important indicator to predict individual health level. Generally, individuals' comprehensive health increases with the level of satisfaction that have toward their current life state (Chapman et al., 2019). Physical exercise is one of the main ways for college students to maintain their physical and mental health. It refers to the physical activities with certain intensity, frequency, and time that individuals carry out in their spare time for the main purpose of health (Song, 2001). Many studies have shown that physical exercise has a positive protective effect on the level of individual cardiopulmonary level, muscle tissue, and brain cognition, and has a good therapeutic effect on metabolic diseases (Sarmento et al., 2017; Leal et al., 2018; Hernandez et al., 2019; 
Borges et al., 2020). So does physical exercise affect life satisfaction? Although few studies have directly examined the relationship between physical exercise and life satisfaction, a number of studies have examined the positive effects of physical exercise on mental health. For example, moderate physical exercise can promote the improvement of individual psychological regulation ability, reduce the level of depression and anxiety, and can significantly improve the individual's quality of life (Kvam et al., 2016; Frederiksen et al., 2021; Nguyen et al., 2021). When individuals are physically healthy, emotionally sound and have a high quality of life, they may also be more satisfied with their current life. In other words, physical activity may be an important factor in life satisfaction. Does physical activity affect life satisfaction through other factors?

We hypothesized that core self-evaluation may be an important mediator variable. Core self-evaluation is an integrated personality trait that includes self-esteem, control point, neuroticism, and general self-efficacy and constitutes a basic self-evaluation (Judge and Bono, 2001). For one thing, physical exercise may have an impact on core self-evaluation. A previous study conducted a self-report questionnaire survey on 326 adolescents and found that cultivating an active lifestyle through physical exercise promotes self-esteem more than reducing bad habits (Pazzaglia et al., 2020). Self-esteem is expressed by individuals through attitudes, language, and behaviors and through subjective evaluation of their own abilities, values, and meanings. Through physical exercise, college students can not only improve their physical fitness and enhance their satisfaction with their body self-image, but also gain friendship and gain recognition from others in the process of physical exercise, and then improve their self-esteem. And self-esteem is an important part of core self-evaluation, so physical exercise can also improve the level of core self-evaluation of college students (Ma and Liu, 2015). For another, core self-evaluation can have an impact on life satisfaction. For example, in a study of 319 students aged 17 to 21 , the results showed that emotional intelligence and core self-evaluation explained $34 \%$ of the difference in life satisfaction, indicating that when adolescents' core self-evaluation increased, their life satisfaction also increased (Esin et al., 2016). Other researchers verified that core self-evaluation is an important predictor of life satisfaction and further revealed its internal mechanism (Zhou and Xuan, 2015). In conclusion, both physical exercise and core self-evaluation can affect life satisfaction, while physical exercise can predict core self-evaluation. Therefore, core self-evaluation may play an important intermediary role between physical exercise and life satisfaction.

We hypothesized that positive emotion might be another important mediator. Positive emotion is short-term experiences that can cause changes in people's thoughts, actions, and psychological reactions (Fredrickson and Branigan, 2005). Research on physical exercise and positive emotion shows that physical exercise not only helps improve individual muscle stiffness and promote metabolism but also can effectively relieve individual stress and increase individual positive emotional performance (Brett et al., 2016; Aguirre-Loaiza et al., 2019). In terms of the relationship between positive emotion and life satisfaction, researchers found that positive emotion had a significant predictive effect on life satisfaction, and school students with higher positive emotional experiences also experienced higher life satisfaction (Datu and King, 2016). Emotions are an indispensable part of an individual's daily life experience. College students who often express positive emotional experiences will develop positive and optimistic attitudes. Even if they encounter negative life events, they are more likely to adopt positive coping methods to deal with problems, enhance the acceptability of negative events, and thus improve the level of life satisfaction (Jiang et al., 2021). It means that physical exercise can improve an individual's life satisfaction by promoting the expression of positive emotion. Therefore, positive emotion may also be an important mediator between physical exercise and life satisfaction.

Although the above analysis shows that core self-evaluation and positive emotion may mediate the relationship between college students' physical exercise and life satisfaction, this study holds that they do not simply play an independent mediating role but may also have a chain mediation effect. In the definition of core self-evaluation proposed by Judge, general self-efficacy is an important part of core self-evaluation. General self-efficacy consists of four aspects, namely, performance achievement, alternative experience, verbal persuasion, and physiological state (Bandura, 1977). In a study looking at what parents can do to promote healthy development in their children, it was found that when parents help children develop higher levels of general self-efficacy, children will show more positive emotion. Individuals' general self-efficacy is an important cognitive resource. Individuals with low selfefficacy tend to overestimate the difficulty of the problem when completing tasks, which leads to anxiety and a higher level of psychological pressure and negative emotion. In contrast, when individuals with high self-efficacy face pressure due to highly challenging events, a high level of self-efficacy can stimulate their self-motivation to overcome difficulties, effectively help them relieve the negative impact caused by stress, and increase their positive and optimistic emotions. $\mathrm{Fu}$ and Tremayne found a significant relationship between healthy behaviors, self-efficacy, and positive emotion. The stronger the sense of self-efficacy, the more healthy behaviors and the more significant positive emotion expressed ( $\mathrm{Fu}$ and Tremayne, 2021). Students with high self-efficacy generally exhibit more healthy behaviors, which is conducive to developing healthy living habits, promotes improvements in life quality, and thus is more conducive to positive emotional experiences (Hascher and Hagenauer, 2016). Therefore, we speculate that core self-assessment can influence life satisfaction through positive emotion.

In summary, this study constructed a chain mediation model between physical exercise and life satisfaction, in order to explore the relationship between physical exercise and life satisfaction and its internal mechanism. Thus, we hypothesized that as:

Hypothesis 1: Physical exercise would be positively associated with core self-evaluation, positive emotion, and life satisfaction. 
Hypothesis 2: In the influence mechanism of college students' physical exercise on life satisfaction, the core self-evaluation and positive emotion play a mediating role, respectively, and there is a chain mediating effect from core self-evaluation to positive emotion.

\section{MATERIALS AND METHODS}

\section{Participants and Procedures}

Participants were undergraduate college students recruited from two colleges and universities (one key and one regular) in western China. We adopted a cluster random sampling method and selected 16 classes (two classes in each grade of two universities) to conduct a questionnaire survey. A total of 860 questionnaires were collected, and 794 valid questionnaires were left after excluding those that were not answered seriously or not standardized. Among them, 429 students are from key universities, with 130, 108, 101, and 90 students from first grade to senior grade, 140 boys, and 289 girls. The other students are from regular universities, with 105, 102, 82, and 76 students from first grade to senior grade, 184 boys, and 181 girls. All the students were aged from 17 to 25 years old $(M=19.96 \pm 1.54)$.

This study was approved by the Research Ethics Committee of Chinese Southwest University. Prior to the study, we contacted the administrators of the participating schools, obtained permission for the questionnaire test and the informed consent of the students themselves. All students participated in the survey voluntarily.

\section{Measures Physical Exercise}

Physical exercise was measured by the Exercise Adherence Questionnaire (Wang et al., 2016). This scale contains 14 items. It measures amateur physical exercise in three dimensions, including effort input (for example, "Regardless of whether I like physical exercise, I will do my best to complete it every time."), emotional experience (for example, "I feel refreshed physically and mentally after exercise."), and behavioral habits (for example, "I have been exercising regularly for at least 6 months."). The scale measures the individual's actual exercise behavior and subjective experience after exercise and has good reliability and validity. Each item was rated on a 5-point scale ( $1=$ totally disagree and $5=$ totally agree), with higher scores indicating more adequate physical exercise. In this study, Cronbach's $\alpha$ was 0.95 for the total scale and ranged from 0.87 to 0.91 for the subscales.

\section{Core Self-Evaluation}

Core self-evaluation was measured by the Core Self-evaluation Scale (Judge and Bono, 2001). The scale has good reliability and validity under the Chinese cultural background ( $\mathrm{Gu}$ and Wen, 2014). The Core Self-evaluation Scale includes
10 items covering four personality traits: self-esteem, control points, neuroticism, and general self-efficacy. A 4-point scale was used for scoring (completely disagree $=1$ and completely agree $=4)$. The Cronbach's $\alpha$ coefficient in this study was 0.85 .

\section{Positive Emotion}

Positive emotion was measured by the positive affect and negative affect scale (PANAS) compiled by Watson (Watson et al., 1988). This scale contains a 10 -item subscale of positive emotion and a 10-item subscale of negative emotion. Each item was rated on a 5 -point scale $(1=$ none of the time and $5=$ most of the time), with higher scores indicating more positive emotional experiences. The PANAS has been shown to have high reliability and validity in the Chinese school environment (Guo and Gan, 2010). The Cronbach's $\alpha$ coefficient in this study was 0.90 .

\section{Life Satisfaction}

Life satisfaction was measured by the Satisfaction with Life Scale compiled (Diener et al., 1985). Individuals evaluate their overall life satisfaction. This scale contains 5 items measured using a 7-point rating scale from 1 (completely nonconformance) to 7 (completely conforming), with higher scores indicating higher life satisfaction. This scale is suitable for use with adolescents in the Chinese school environment, and the reliability of the whole scale is excellent (Xiong and $\mathrm{Xu}, 2009)$. The Cronbach's $\alpha$ coefficient in this study was 0.85 .

\section{Analytic Strategy}

The study used SPSS 22.0 and the Process plug-in to analyze the data and used Process model 6 to test the chain mediation model. For the significance test of the regression coefficient, the bootstrapping method with 5,000 repeated samples was selected to obtain a robust standard error and a 95\% deviationcorrected confidence interval (CI). When the CI does not contain zero, the effect is significant.

At the beginning of the data analysis, we used Harman's single-factor test to explore whether there may be common method biases in this study. The results showed that there were seven factors with eigenvalues greater than 1; among these, the largest factor explained $32.26 \%$ of the variance, less than the critical range of $40 \%$. Therefore, we believe that the possibility of common method biases in this study is relatively small.

\section{RESULTS}

\section{Descriptive Statistics and Analysis of the Correlations Between Variables}

Descriptive statistics and correlation analysis were conducted for each variable. As shown in Table 1, there was a significant positive correlation between physical exercise, core self-evaluation, positive emotion, and life satisfaction. 
TABLE 1 | Descriptive statistics and interrelations among all observed variables.

\begin{tabular}{|c|c|c|c|c|c|c|}
\hline Variables & $M$ & SD & 1 & 2 & 3 & 4 \\
\hline 1 Physical exercise & 49.78 & 11.66 & - & & & \\
\hline 2 Core self-evaluation & 33.49 & 6.20 & $0.25 * * *$ & - & & \\
\hline 3 Positive emotion & 33.04 & 6.60 & $0.54 * * *$ & $0.41 * * *$ & - & \\
\hline 4 Life satisfaction & 22.27 & 5.47 & $0.35 * * *$ & $0.39 * * *$ & $0.54 * * *$ & - \\
\hline
\end{tabular}

$* * * p<0.001$

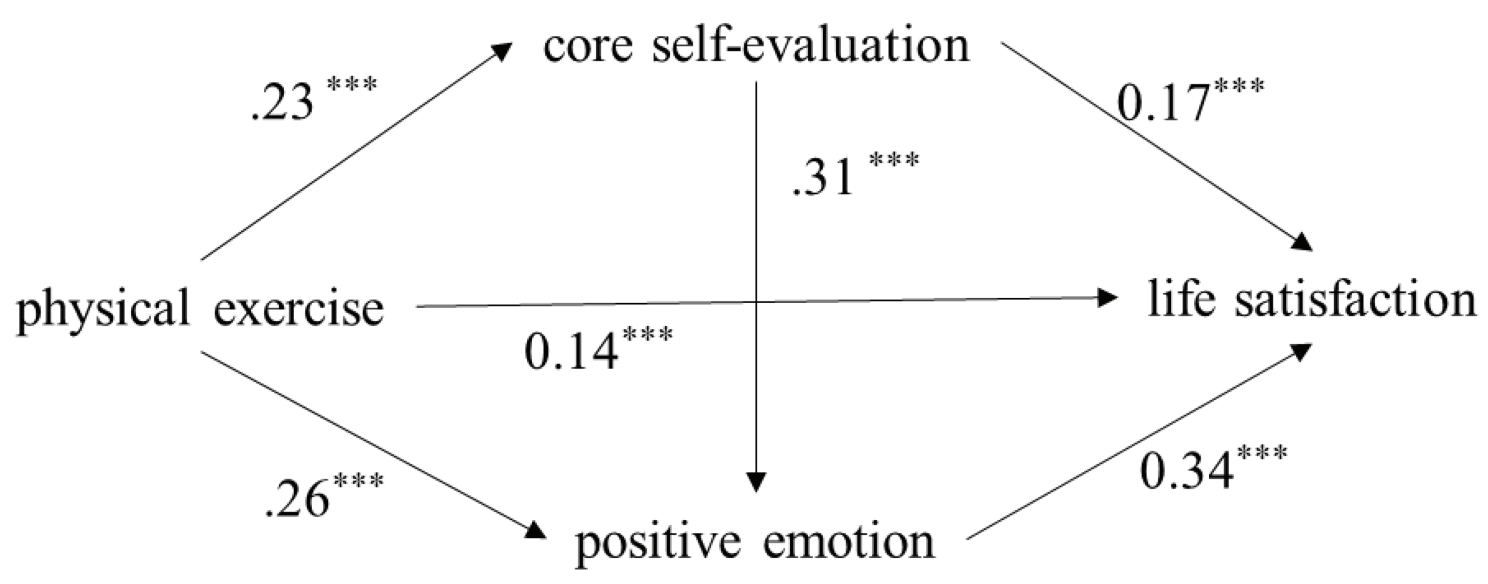

FIGURE 1 | Model of the mediator role of core self-evaluation and positive emotion in the relationship between physical exercise and life satisfaction. *** $p<0.001$, Significant regression coefficient.

\section{Testing the Mediating Effects of Core Self- Evaluation and Positive Emotion}

Taking physical exercise as the independent variable, life satisfaction as the dependent variable, and core self-evaluation and positive emotion as the mediating variables, the chain mediation effect test yielded the results shown in Figure 1 and Tables 2, 3.

The results show that, firstly, there are significant positive correlations between physical exercise, core self-evaluation, positive emotions, and life satisfaction, which supports Hypothesis 1. Secondly, physical exercise has a significant indirect effect on life satisfaction through core self-evaluation $(\beta=0.039,95 \% \mathrm{CI}=[0.01,0.04])$, that is, core self-evaluation has a significant mediating effect on the relationship between physical exercise and life satisfaction. Thirdly, physical exercise has a significant indirect effect on life satisfaction through positive emotions $(\beta=0.084,95 \% \mathrm{CI}=[0.06,0.12])$, that is, positive emotions have a significant mediating effect on the relationship between physical exercise and life satisfaction. Finally, the indirect effect of physical exercise on life satisfaction through core self-evaluation and positive emotion was significant $(\beta=0.024,95 \% \mathrm{CI}=[0.01,0.02])$. In other words, core selfevaluation can affect positive emotion. At the same time, the chain mediation path from physical exercise to core selfevaluation, then to positive emotion, and finally to life satisfaction is significant, supporting Hypothesis 2. These results indicate that core self-evaluation and positive emotion play a continuous mediating role in the relationship between physical exercise and life satisfaction. The total mediating effect accounted for $51.58 \%$ of the total effect; that is, $51.58 \%$ of the effect of physical exercise on college students' life satisfaction through the two variables of core self-evaluation and positive emotion.

\section{DISCUSSION}

As expected, physical exercise, core self-evaluation, positive emotion, and life satisfaction had significant positive relationships with each other. In addition, we found that core self-evaluation and positive emotion played a mediating role in the relationship between physical exercise and life satisfaction, and the chain mediation effect was significant.

First of all, from the relationship between physical exercise and core self-evaluation, physical exercise can significantly positively predict core self-evaluation. Although no studies have directly examined the relationship between physical exercise and core self-evaluation, existing studies have shown that physical exercise can significantly increase an individual's self-esteem or self-awareness (Mcauley et al., 1997; Wong et al., 2021). In the process of physical exercise, the individual's body will become stronger, which will improve the individual's positive body selfimage. At the same time, individuals can make friends with the same interests, which also provides them with peer support and promotes the improvement of self-esteem. Therefore, core self-evaluation, as a comprehensive reflection of self-esteem and 


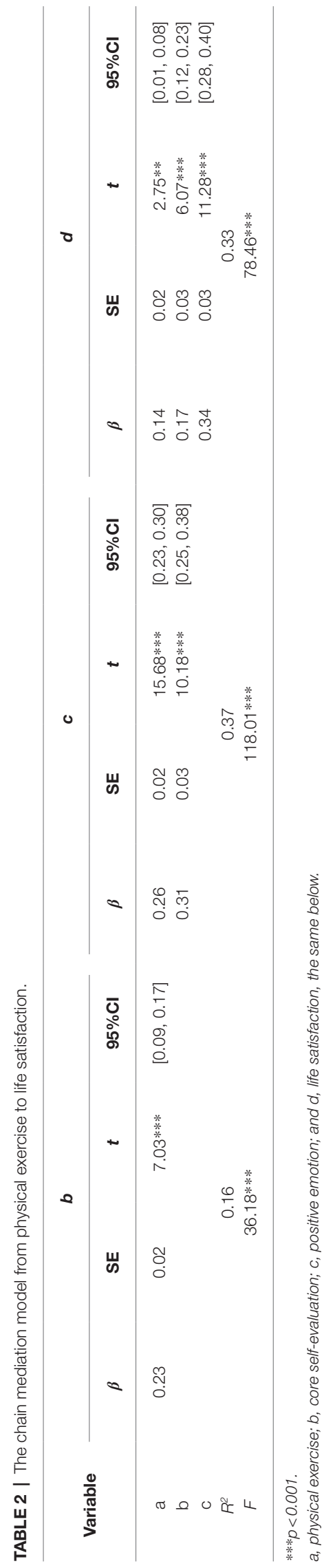

TABLE 3 | Standardized indirect effects from physical exercise to life satisfaction.

\begin{tabular}{|c|c|c|c|c|}
\hline Model & $\begin{array}{c}\beta \\
\text { (standardized } \\
\text { indirect effect) }\end{array}$ & SE & $95 \% \mathrm{Cl}$ & $\begin{array}{c}\text { Relative } \\
\text { mediating } \\
\text { effect }\end{array}$ \\
\hline $\begin{array}{l}\text { Total indirect } \\
\text { effect }\end{array}$ & 0.147 & 0.013 & $0.10,0.16$ & $51.58 \%$ \\
\hline$a-b-d$ & 0.039 & 0.006 & $0.01,0.04$ & $13.68 \%$ \\
\hline$a-c-d$ & 0.084 & 0.013 & $0.06,0.12$ & $29.47 \%$ \\
\hline$a-b-c-d$ & 0.024 & 0.003 & $0.01,0.02$ & $8.42 \%$ \\
\hline
\end{tabular}

self-awareness, will also be positively affected by physical exercise. Secondly, physical exercise significantly positively predicted positive emotion was also consistent with prior research (Greenwood, 2019). Physical exercise can promote the body to secrete dopamine, which is closely related to pleasure, thus increasing the individual's positive emotional experience. Additionally, consistent with prior research, physical exercise was positively associated with life satisfaction (Moreno-murcia et al., 2017; Reigal et al., 2019). From the function of physical exercise, moderate physical exercise can enhance the physical quality of students, while maintaining good physical health is the desire of most people. Therefore, it is understandable that moderate physical exercise can improve college students' life satisfaction. In conclusion, the results of this study demonstrate the positive promoting effect of physical exercise on mental health, and also support Hypothesis 1.

As for Hypothesis 2, physical exercise can directly affect life satisfaction and can also affect life satisfaction through the mediation of core self-evaluation and positive emotion. From the perspective of core self-evaluation, physical exercise can improve core self-evaluation. Individuals with higher core self-evaluation have stronger psychological adjustment ability, are more likely to use positive coping strategies, and have higher life satisfaction (Zhao and Shi, 2018). Therefore, core self-evaluation can play an important role in the bridge between physical and mental health, which is consistent with the existing findings (Xiang et al., 2019). From the perspective of positive emotion, physical exercise can also promote the production of individual positive emotion. When individuals experience more positive emotion, the proportion of negative emotions is significantly reduced, which helps individuals form positive and optimistic attitudes, have a greater sense of hope, and show higher life satisfaction (Ligeza et al., 2019; Bo et al., 2020). The results also showed that the mediation path of positive emotion has the greatest influence on the total mediation effect, indicating that the influence of physical exercise on life satisfaction is largely played by positive emotion. Moreover, we also found that core self-evaluation and positive emotions play a chain mediating role in the impact of physical exercise on college students' life satisfaction. In other words, core selfevaluation affects life satisfaction by influencing positive emotion. Although there are relatively few studies that directly examine the relationship between core self-evaluation and positive emotions, many studies have found that the improvement of core self-evaluation will improve the level of self-esteem, enhance 
the experience of positive emotions, and adopt a more positive way to face difficulties and challenges, thus improving subjective wellbeing and work efficiency (Kim et al., 2016; Ding and Lin, 2020; Firouznia et al., 2021). This study directly proves that the improvement of core self-evaluation can enhance individuals' positive emotional experience, and further proves the importance of core self-evaluation in individuals' positive mental health.

In general, through physical exercise, individuals can improve their physical quality, gain friendship, enhance self-confidence, and thus improve their core self-evaluation. Meanwhile, in this process, individuals can enhance their positive emotional experience and ultimately improve their life satisfaction. This enlightens us, physical health and mental health are closely linked, we should encourage students to take an active part in physical exercise activities, cultivate a strong physique, promote healthy body, also need to guide students to establish positive self-evaluation, cultivate a positive, optimistic attitude toward life, and try to sports activities and the organic integration of mental health education.

\section{LIMITATIONS AND FUTURE DIRECTIONS}

This study also has some deficiencies. First, due to the limitation of objective factors, such as time and research funds, this study adopted a cross-sectional study design. Although existing studies have provided a solid foundation for this study, the results of this study can be enriched and expanded through further follow-up and empirical studies. Second, the subjects of this study were all college students without distinction. In future research, we can further explore the impact of physical exercise on life satisfaction of people of different ages. Third, physical exercise in this study was self-reported by participants through questionnaires. In the future, we can further explore the influence of frequency, time, and form of physical exercise on life satisfaction.

\section{CONCLUSION}

In conclusion, this study investigates how college students' physical exercise influences life satisfaction. Specifically, we found that physical exercise significantly positively predicted life satisfaction and verified the mediating role of core self-evaluation and positive emotions in this relationship. The results also

\section{REFERENCES}

Aguirre-Loaiza, H., Arenas, J., Arias, I., Franco-Jimenez, A., Barbosa-Granados, S., Ramos-Bermudez, S., et al. (2019). Effect of acute physical exercise on executive functions and emotional recognition: analysis of moderate to high intensity in young adults. Front. Psychol. 10:2774. doi: 10.3389/ fpsyg.2019.02774

Bandura, A. (1977). Self-efficacy: Toward a unifying theory of behavioral change. Psychol. Rev. 84, 191-215. doi: 10.1037/0033-295X.84.2.191

Bo, X., Xia, B., Ma, Z., and Hu, Y. Y. (2020). Research on the relationship between physical exercise, psychological flexibility and positive emotion of college students based on computer mathematical model. J. Phys. Conf. Ser. 1578:012009. doi: 10.1088/1742-6596/1578/1/012009 confirmed a chain mediation model between physical exercise, core self-evaluation, positive emotions, and life satisfaction.

\section{DATA AVAILABILITY STATEMENT}

The raw data supporting the conclusion of this article will be made available by the authors, without undue reservation.

\section{ETHICS STATEMENT}

The studies involving human participants were reviewed and approved by the Research Ethics Committee of Southwest University. Written informed consent to participate in this study was provided by the participants' legal guardian/next of kin.

\section{AUTHOR CONTRIBUTIONS}

On the basis of reading related literature, FL raised the questions of this research and was responsible for making research plans, collecting data, and writing articles during the whole research process. ZZ made a great contribution to the data collection and analysis of the research and the revision of the paper. BJ was mainly responsible for the supervision and guidance of the whole research process and provided the necessary financial and personnel assistance. All authors contributed to the article and approved the submitted version.

\section{FUNDING}

This research has been supported by the Key Project of Chinese Ministry of Education during the 14th Five-Year Plan Period (Title: Research on the bottleneck and collaborative governance of the construction of Chinese campus football characteristic schools in the new era; Serial number: DLA210371).

\section{ACKNOWLEDGMENTS}

We thank all the participants in this study.

Borges, M. V. D., Medeiros, J. F. P., de Sousa, E. C., da Costa, J. R. R., Lemos, T. M. A. M., Dantas, P. M. S., et al. (2020). Metabolic profile and body composition in twins concordant and discordant for physical exercise. Twin Res. Hum. Genet. 23, 241-246. doi: 10.1017/thg.2020.58

Brett, L., Traynor, V., and Stapley, P. (2016). Effects of physical exercise on health and well-being of individuals living with a dementia in nursing homes: A systematic review. J. Am. Med. Dir. Assoc. 17, 104-116. doi: 10.1016/j.jamda.2015.08.016

Chapman, A., Fujii, H., and Managi, S. (2019). Multinational life satisfaction, perceived inequality and energy affordability. Nat. Sustain. 2, 508-514. doi: 10.1038/s41893-019-0303-5

Datu, J. A. D., and King, R. B. (2016). Prioritizing positivity optimizes positive emotion and life satisfaction: A three-wave longitudinal study. Pers. Indiv. Differ. 96, 111-114. doi: 10.1016/j.paid.2016.02.069 
Dew, T., and Huebner, E. (1994). Adolescents perceived quality of life: An exploratory investigation. J. School Psychol. 32, 185-199. doi: 10.1016/0022-4405(94)90010-8

Diener, E., Emmons, R. A., Larsen, R. J., and Griffin, S. (1985). The satisfaction with life scale. J. Pers. Assess. 49, 71-75. doi: 10.1207/ s15327752jpa4901_13

Ding, H., and Lin, X. (2020). Exploring the relationship between core selfevaluation and strengths use: The perspective of emotion. Pers. Indiv. Differ. 157:109804. doi: 10.1016/j.paid.2019.109804

Esin, Ö., Erdal, H., and Deniz, M. E. (2016). Emotional intelligence, core-self evaluation, and life satisfaction. Psychology 7, 143-145. doi: 10.4236/ psych.2016.72017

Firouznia, M., Hosseini, S. H., and Karamabad, M. M. M. (2021). Affectivecognitive nature of engagement: correlating psychological capital and coreself-evaluations to work engagement via positive affects. Int. J. Procurement Management 14, 213-229. doi: 10.1504/IJPM.2021.113492

Frederiksen, K. P., Stavestrand, S. H., Venemyr, S. K., Sirevag, K., and Hovland, A. (2021). Physical exercise as an add-on treatment to cognitive behavioural therapy for anxiety: A systematic review. Behav. Cogn. Psychoth. 49, 626-640. doi: $10.1017 /$ S1352465821000126

Fredrickson, B. L., and Branigan, C. (2005). Positive emotion broaden the scope of attention and thought-action repertoires. Cognition Emontion. 19, 313-332. doi: 10.1080/02699930441000238

Fu, K. W., and Tremayne, K. S. (2021). Self-efficacy and self-control mediate the relationship between negative emotions and attitudes toward plagiarism. J. Acad. Ethics., 1-21. doi: 10.1007/s10805-021-09415-3

Greenwood, B. N. (2019). The role of dopamine in overcoming aversion with exercise. Brain Res. 1713, 102-108. doi: 10.1016/j.brainres.2018.08.030

$\mathrm{Gu}, \mathrm{H}$., and Wen, Z. (2014). The impact of the wording effect on reliability and criterion validity of self-report questionnaires: The case of the core self-evaluation scale. J. Psychological Sci. 37, 1245-1252. doi: 10.16719/j. cnki.1671-6981.2014.05.027

Guo, M., and Gan, Y. (2010). Reliability and validity of the Chinese version of positive and negative affect schedule-expanded in 660 college students. Chinese Ment. Health J. 24, 524-528. doi: 10.3969/j. issn.1000-6729.2010.07.012

Hascher, T., and Hagenauer, G. (2016). Openness to theory and its importance for pre-service teachers' self-efficacy, emotions, and classroom behaviour in the teaching practicum. Int. J. Educ. Res. 77, 15-25. doi: 10.1016/j.ijer.2016.02.003

Hernandez, M. A., Reigal, R. E., Lopez, W. J., Serpa, S., Samdal, O., Morales, S. V., et al. (2019). Physical activity, sports practice, and cognitive functioning: The current research status. Front. Psychol. 10:2658. doi: 10.3389/ fpsyg.2019.02658

Jiang, X., Ji, L., Chen, Y., Zhou, C., Ge, C., and Zhang, X. (2021). How to improve the well-being of youths: an exploratory study of the relationships among coping style, emotion regulation, and subjective well-being using the random forest classification and structural equation modeling. Front. Psychol. 12:637712. doi: 10.3389/fpsyg.2021.637712

Judge, T. A., and Bono, J. E. (2001). Relationship of core self-evaluations traitsself-esteem, generalized self-efficacy, locus of control, and emotional stabilitywith job satisfaction and job performance: A meta-analysis. J. Appl. Psychol. 86, 80-92. doi: 10.1037/0021-9010.86.1.80

Kim, H., Schimmack, U., Cheng, C., Webster, G. D., and Spectre, A. (2016). The role of positive self-evaluation on cross-cultural differences in wellbeing. Cross-Cult. Res. 50, 85-99. doi: 10.1177/1069397115617902

Kvam, S., Kleppe, C. L., Nordhus, I. H., and Hovland, A. (2016). Exercise as a treatment for depression: A meta-analysis. J. Affect. Disord. 202, 67-86. doi: $10.1016 /$ j.jad.2016.03.063

Leal, L. G., Lopes, M. A., and Batista, M. L. (2018). Physical exercise-induced myokines and muscle-adipose tissue crosstalk: A review of current knowledge and the implications for health and metabolic diseases. Front. Physiol. 9:1307. doi: $10.3389 /$ fphys.2018.01307

Ligeza, T. S., Kalamala, P., Tarnawczyk, O., Maciejczyk, M., and Wyczesany, M. (2019). Frequent physical exercise is associated with better ability to regulate negative emotions in adult women: The electrophysiological evidence. Ment. Health Phys. Act. 17:100294. doi: 10.1016/j.mhpa.2019.100294
Ma, A., and Liu, S. (2015). The relationship among physical exercise, physical self-esteem and general self-efficacy of college students. Sport Sci. Tech. 36, 57-61. doi: 10.14038/j.cnki.tykj.2015.03.022

Mcauley, E., Mihalko, S. L., and Bane, S. M. (1997). Exercise and self-esteem in middle-aged adults: multidimensional relationships and physical fitness and self-efficacy influences. J. Behav. Med. 20, 67-83. doi: 10.1023/A:1025591214100

Moreno-murcia, J. A., Belando, N., Huescar, E., and Torres, M. D. (2017). Social support, physical exercise and life satisfaction in women. Rev. Lat. Am. Psicol. 49, 194-202. doi: 10.1016/j.rlp.2016.08.002

Nguyen, T. M., Nguyen, V. H., and Jin, H. K. (2021). Physical exercise and health-related quality of life in office workers: a systematic review and metaanalysis. Int. J. Env. Res. Pub. He. 18:3791. doi: 10.3390/ijerph18073791

Pazzaglia, F., Moè, A., Cipolletta, S., Chia, M., Galozzi, P., Masiero, S., et al. (2020). Multiple dimensions of self-esteem and their relationship with health in adolescence. Int. J. Env. Res. Pub. He. 17:2616. doi: 10.3390/ijerph17082616

Reigal, R. E., Barrero, S., Martin, I., Morales-Sanchez, V., de Mier, R. J. R., Hernandez-Mendo, A., et al. (2019). Relationships between reaction time, selective attention, physical activity and physical fitness in children. Front. Psychol. 10:2278. doi: 10.3389/fpsyg.2019.02278

Sarmento, A. D., Santos, A. D., Trombetta, I. C., Dantas, M. M., Marques, A. C. O., do Nascimento, L. S., et al. (2017). Regular physical exercise improves cardiac autonomic and muscle vasodilatory responses to isometric exercise in healthy elderly. Clin. Interv. Aging 12, 1021-1028. doi: 10.2147/CIA.S120876

Song, X. (2001). Research on factors related to exercise behavior. J. Chengdu Phys. Educ. Inst. 27, 49-52. doi: 10.3969/j.issn.1001-9154.2001.02.015

Wang, S., Liu, Y., and Gu, C. (2016). Influential mechanism of amateur sport group cohesiveness on individual's exercise adherence: A regulatory two-layer intermediary model. J. Wuhan Inst. Phys. Edu. 50, 73-80. doi: 10.3969/j. issn.1000-520X.2016.03.012

Watson, D., Clark, L. A., and Tellegen, A. (1988). Development and validation of brief measures of positive and negative affect: The panas scales. J. Pers. Soc. Psychol. 54, 1063-1070. doi: 10.1037/0022-3514.54.6.1063

Wong, M., Chung, P. K., and Leung, K. M. (2021). Examining the exercise and self-esteem model revised with self-compassion among Hong Kong secondary school students using structural equation modeling. Int. J. Environ. Res. Public Health 18:3661. doi: 10.3390/ijerph18073661

Xiang, Y., Zhao, J., Li, Q., Zhang, W., Dong, X., and Zhao, J. (2019). Effect of core self-evaluation on mental health symptoms among Chinese college students: The mediating roles of benign and malicious envy. Psychiat. Ann. 49, 277-284. doi: 10.3928/00485713-20190508-01

Xiong, C., and Xu, Y. (2009). Reliability and validity of the satisfaction with life scale for Chinese demos. China J. Health Psychol. 17, 948-949. doi: 10.13342/j.cnki.cjhp.2009.08.026

Zhao, X. J., and Shi, C. X. (2018). The relationship between regulatory emotional self-efficacy and core self-evaluation of college students: The mediation effects of suicidal attitude. Front. Psychol. 9:598. doi: 10.3389/fpsyg.2018.00598

Zhou, J., and Xuan, J. (2015). Core self-evaluation and life satisfaction: The person-environment fit perspective. Pers. Indiv. Differ. 75, 68-73. doi: 10.1016/j. paid.2014.11.013

Conflict of Interest: The authors declare that the research was conducted in the absence of any commercial or financial relationships that could be construed as a potential conflict of interest.

Publisher's Note: All claims expressed in this article are solely those of the authors and do not necessarily represent those of their affiliated organizations, or those of the publisher, the editors and the reviewers. Any product that may be evaluated in this article, or claim that may be made by its manufacturer, is not guaranteed or endorsed by the publisher.

Copyright (C) $2021 \mathrm{Liu}, \mathrm{Zhu}$ and Jiang. This is an open-access article distributed under the terms of the Creative Commons Attribution License (CC BY). The use, distribution or reproduction in other forums is permitted, provided the original author(s) and the copyright owner(s) are credited and that the original publication in this journal is cited, in accordance with accepted academic practice. No use, distribution or reproduction is permitted which does not comply with these terms. 